\title{
Cardiac valve calcification: an immutable pathologic finding in chronic kidney disease?
}

\author{
Antonio Bellasi ${ }^{1}$, Andrea Galassi ${ }^{2}$, Sergio Papagni ${ }^{3}$, \\ Mario Cozzolino ${ }^{4}$
}

\author{
${ }^{1}$ Department of Nephrology, Ospedale Sant'Anna-Como, \\ Como - Italy \\ ${ }^{2}$ Department of Nephrology, Azienda di Desio e Vimercate, \\ Desio - Italy \\ ${ }^{3}$ Division of Nephrology, Centro Dialisi CBH-Città di \\ Bisceglie, Città di Bisceglie - Italy \\ ${ }^{4}$ Department of Health Sciences, University of Milan, \\ Milan - Italy
}

\begin{abstract}
Although less frequent than vascular calcification, cardiac valve calcification (CVC) is a relevant clinical problem affecting about $2 \%-10 \%$ of adults from the general population aged $\mathbf{7 5}$ years and older, and is 5- to 10-fold more prevalent in individuals with impaired kidney function. An expanding body of evidence suggests that mineral metabolism abnormalities aside from traditional cardiovascular risk factors are involved in CVC pathogenesis. Nonetheless, very few studies have investigated whether mineral metabolism manipulation impacts CVC. In this issue of the Journal of Nephrology, it is reported that a combination of low-phosphate diet and sevelamer may reduce CVC. Though the observational nature of that study and the lack of a control group significantly limit the generalizability of these results, they fit in with the ongoing debate on the role of chronic kidney disease mineral bone metabolism (CKD-MBD) in the pathogenesis of vascular disease and suggest the importance of mineral metabolism control in patients with CKD.
\end{abstract}

Key words: CKD-MBD, Low-phosphate diet, Sevelamer, Valvular calcification

Although less frequent than vascular calcification, cardiac valve calcification is a relevant clinical problem in the general population as well as in patients with chronic kidney disease (CKD) (1-3). It is estimated that about $2 \%-10 \%$ of adults 75 years of age and older exhibit aortic and mitral valve calcification $(3,4)$. A significant 5 - to 10 -fold greater prevalence is reported in age- and sex-matched individuals with impaired kidney function $(1,5,6)$.

Although the pathologic mechanisms are only partially understood (7), some evidence suggests that heart valvular calcification shares similar risk factors and pathogenetic features with atherosclerosis (older age, male sex, hypertension, smoking, hypercholesterolemia and diabetes) (8). However, mineral bone metabolism and other nontraditional cardiovascular (CV) risk factors have also been shown to be involved $(6,9,10)$ and may partially explain why as many as $50 \%$ of subjects from the general population with valvular calcification do not have clinically meaningful atherosclerosis (11), and why the risk of cardiac valve calcification increases as glomerular filtration (GFR) declines $(5,9)$. In particular, observational studies suggest a strong and independent association between serum phosphorous levels and the risk of valve calcification (6). In the Multi-Ethnic Study of Atherosclerosis, serum phosphorus levels were associated with aortic and mitral valve calcification in a cohort of 439 subjects with mild-to-moderate CKD (mean estimated GFR [eGFR] about $50 \mathrm{ml} / \mathrm{min}$ per $1.73 \mathrm{~m}^{2}$ ) (6). Each $1 \mathrm{mg} / \mathrm{dL}$ increase in serum phosphate corresponded to a significant $25 \%$ and $62 \%$ greater prevalence of aortic valve and mitral valve calcification, respectively (6). Notably, adjustment for eGFR, age and other parameters of mineral metabolism did not substantially affect these associations, which were significant even for serum phosphate levels within the reference range (6).

Irrespective of the pathogenesis, heart valve calcifications predict poor survival in both the general and the CKD population $(2,3,10,12,13)$. Indeed, calcified valves have 
been associated with extensive calcification burden in the coronary arteries $(1,14)$, carotid atherosclerosis and calcification (15), progressive valve narrowing, left ventricular hypertrophy and heart failure $(10,16)$. In a group of 140 transplant candidates with late stages of CKD or on maintenance dialysis, evidence of calcified mitral annulus was significantly associated with left atrial and ventricular enlargement, cardiac mass index, left ventricular diastolic dysfunction and mortality (10). In a series of 144 prevalent end-stage renal disease (ESRD) patients, the presence and the extension of aortic and mitral valve calcification assessed via cardiac computed tomography (CT) predicted the risk of all-cause mortality independently of several confounders. Notably, a combined CT score of valvular and coronary artery calcification (CAC) was strongly associated with all-cause mortality during follow-up (hazard ratio for highest vs. lowest tertile, 2.21; 95\% confidence interval, 1.08-4.54) (2).

Due to the fact that the underlying pathophysiology remains incompletely defined and there is a lack of effective medical treatments capable of altering the natural course of cardiac valve calcification, whether aortic or mitral valve calcifications represent a marker or a promoter of $\mathrm{CV}$ disease still needs to be elucidated. Nonetheless, in light of how easy it is to make a cardiac valve calcification assessment, this information could be of use in risk stratification and potentially to guide treatment individualization (17).

In this regard, in the current issue of the Journal of Nephrology an interesting observation is reported. In a single-center, single-arm, prospective study, the impact on valvular calcification of sevelamer $\mathrm{HCl}$ in combination with a low-protein diet was tested in 160 consecutive CKD stage 3 and 4 patients. Of note, all recruited subjects at study inception had evidence of either mitral or aortic valve calcification as well as suboptimal serum phosphate control with median levels of $4.9 \mathrm{mg} / \mathrm{dL}$ (interquartile range 4.7-5.2). After 12 months of treatment, a significant reduction in aortic and mitral valve calcification burden as assessed via a semiquantitative echocardiographic score was noted. This finding was coupled with a significant reduction in phosphate balance as demonstrated by the lower levels of serum fibroblast growth factor 23 (FGF23) and phosphate as well as 24-hour urinary phosphate excretion.

Though the lack of a control group and the observational nature of the study preclude definitive causal conclusions, these results further corroborate the hypothesis that phosphorous and mineral metabolism manipulation may represent a potential therapeutic target in CKD patients with evidence of CV calcification (17).
Two other studies have investigated the impact of different agents of valvular calcification progression. In a post hoc analysis of the Treat to Goal study (18), Raggi and coworkers observed in a cohort of 132 prevalent hemodialysis patients that 52 weeks of sevelamer $\mathrm{HCl}$ use was associated with a significant attenuation of cardiac calcification when compared with calcium salts (18). In particular, the authors reported a significantly greater number of sevelamer-treated subjects experiencing an arrest ( $45 \%$ vs. $28 \%, p=0.04)$ or regression ( $26 \%$ vs. $10 \%, p=0.02)$ in total valvular and vascular calcification (18). In a more recent study, the impact of cinacalcet plus low-dose vitamin $D$ vs. flexible doses of vitamin $\mathrm{D}$, on cardiac calcification was tested (19). As highlighted in a subsequent post hoc analysis, prevalent hemodialysis subjects with evidence of aortic valve calcification were likely to benefit from the combined treatment with cinacalcet and low doses of vitamin $\mathrm{D}$ and experience significant cardiac valve calcification progression attenuation (20).

Hence, there is evidence supporting the notion that mineral metabolism is implicated in the pathogenesis of vascular calcification. The current article adds to the existing body of evidence suggesting that a precocious intervention (i.e., at CKD stage 3B) aimed at lowering of phosphate balance could result in CV disease reduction in CKD. When and how to correct these abnormalities in the course of CKD still remains debatable $(21,22)$. Observational data support the notion that the risk of cardiac calcification (6) as well as CV death (23) and all-cause mortality (23) is increased for serum phosphate within the reference range. Nonetheless, we currently lack a validated marker of phosphate metabolism imbalance to discriminate those individuals at increased risk for CV events. Indeed, phosphaturia and phosphoremia (with the exceptions of extreme levels) only weakly correlate with phosphate balance, and newer markers such as FGF23) (24) or Klotho are not routinely assessed (21). Far more importantly, the complicated interplay of different factors modulates the risk to which an individual is exposed (25). In a large cohort of 872 subjects recruited in the Heart and Soul study, phosphaturia modulated the risk associated with high phosphate balance (25). Individuals with high levels of FGF-23 but preserved phosphaturic response experienced a lower risk of both all-cause mortality and CV events when compared with individuals with high FGF-23 and low urinary phosphate output (25).

In light of the findings that complete FGF-23 neutralization is associated with increased risk of death in an animal model (26), it is plausible that phosphate balance is far more important than serum phosphate per se, showing that phosphate dampening is, to a certain degree, an adaptive mechanism to prevent phosphorous accumulation (27). 
These hypotheses may potentially explain the surprising results of 2 recent randomized controlled trials that have failed to show a positive effect of sevelamer carbonate vs. placebo on cardiac mass (28), arterial stiffness (28), left ventricular function (28) and CAC (29) in mild-to-moderate CKD with normal serum levels. Aside from study design differences (21), these results are in contrast to previous findings that showed a significant improvement associated with sevelamer use, with regard to endothelial function (30, 31), CAC (32) and survival (32) in moderate CKD individuals with hyperphosphatemia. However, as in the study presented in this issue of the Journal of Nephrology (33), the inclusion of individuals with overt hyperphosphatemia may have selected those individuals with more severe phosphorous imbalances, uncompensated by phosphaturic hormones and likely more susceptible to phosphate-lowering interventions. Finally, the use of calcium-containing phosphate binders instead of placebo may also contribute to explaining these results (34). Indeed, a growing body of evidence suggests a detrimental effect of calcium supplementation in normal kidney function subjects (35-37), enhanced positive calcium balance (38) in CKD and a potential for FGF23 increase (39) that in turn worsens mineral metabolism homeostasis.
In summary, current results fit in with the ongoing debated on the role of chronic kidney disease mineral bone metabolism (CKD-MBD) in the pathogenesis of vascular disease in CKD. In light of the noninvasive and inexpensive techniques available to screen for valvular calcification, future research should test, in a prospective and randomized study, whether sevelamer and a low-phosphate diet can attenuate or reverse valvular calcification, and whether this intervention improves CKD patients' survival.

Financial support: None.

Conflicts of interest: A.B. has received speaking honoraria from Genzyme, Sanofi, Amgen and Sanifit. A.G. has received speaking honoraria form AbbVie and Shire. M.C. has received speaking honoraria form AbbVie, Sanofi and Amgen.

Address for correspondence:

Antonio Bellasi

Department of Nephrology

Ospedale Sant'Anna-Como

Via Ravona

IT-22020 Como, Italy

antoniobellasi@gmail.com

\section{References}

1. Bellasi A, Ferramosca E, Ratti C, et al. Cardiac valve calcification is a marker of vascular disease in prevalent hemodialysis patients. J Nephrol. 2012;25:211-218.

2. Raggi P, Bellasi A, Gamboa C, et al. All-cause mortality in hemodialysis patients with heart valve calcification. Clin J Am Soc Nephrol. 2011;6:1990-1995.

3. Blaha MJ, Budoff MJ, Rivera JJ, et al. Relation of aortic valve calcium detected by cardiac computed tomography to allcause mortality. Am J Cardiol. 2010;106:1787-1791.

4. Nkomo VT, Gardin JM, Skelton TN, et al. Burden of valvular heart diseases: a population-based study. Lancet. 2006; 368:1005-1011.

5. Ribeiro S, Ramos A, Brandão A, et al. Cardiac valve calcification in haemodialysis patients: role of calcium-phosphate metabolism. Nephrol Dial Transplant. 1998;13(8):2037-2040.

6. Adeney KL, Siscovick DS, Ix JH, et al. Association of serum phosphate with vascular and valvular calcification in moderate CKD. J Am Soc Nephrol. 2009;20:381-387.

7. Cozzolino M, Brenna I, Ciceri P, et al. Vascular calcification in chronic kidney disease: a changing scenario. J Nephrol. 2011;24(Suppl 18):S3-S10.

8. Miller JD, Weiss RM, Heistad DD. Calcific aortic valve stenosis: methods, models, and mechanisms. Circ Res. 2011; 108:1392-1412.

9. Mazzaferro S, Coen G, Bandini S, et al. Role of ageing, chronic renal failure and dialysis in the calcification of mitral annulus. Nephrol Dial Transplant. 1993;8:335-340.

10. Sharma R, Pellerin D, Gaze DC, et al. Mitral annular calcification predicts mortality and coronary artery disease in end stage renal disease. Atherosclerosis. 2007;191:348-354.

11. Qian J, Chen Z, Ge J, et al. Relationship between aortic valve calcification and the severity of coronary atherosclerotic disease. J. Heart Valve Dis. 2010;19:466-470.

12. Otto CM, Lind BK, Kitzman DW, Gersh BJ, Siscovick DS. Association of aortic-valve sclerosis with cardiovascular mortality and morbidity in the elderly. N Engl J Med. 1999;341 (3):142-147.

13. Wang AY, Wang M, Woo J, et al. Cardiac valve calcification as an important predictor for all-cause mortality and cardiovascular mortality in long-term peritoneal dialysis patients: a prospective study. J Am Soc Nephrol. 2003;14(1): 159-168.

14. Bellasi A, Ferramosca E, Muntner P, et al. Correlation of simple imaging tests and coronary artery calcium measured by computed tomography in hemodialysis patients. Kidney Int. 2006;70:1623-1628. 
15. Wang AY, Ho SS, Wang M, et al. Cardiac valvular calcification as a marker of atherosclerosis and arterial calcification in endstage renal disease. Arch Intern Med. 2005;165(3):327-332.

16. Dweck MR, Boon NA, Newby DE. Calcific aortic stenosis: a disease of the valve and the myocardium. J Am Coll Cardiol. 2012;60:1854-1863.

17. Kidney Disease: Improving Global Outcomes (KDIGO) CKDMBD Work Group. KDIGO clinical practice guideline for the diagnosis, evaluation, prevention, and treatment of chronic kidney disease-mineral and bone disorder (CKD-MBD). Kidney Int Suppl. 2009 Aug;(113):S1-130.

18. Raggi $P$, Bommer J, Chertow GM. Valvular calcification in hemodialysis patients randomized to calcium-based phosphorus binders or sevelamer. J Heart Valve Dis. 2004;13(1): 134-141.

19. Raggi P, Chertow GM, Torres PU, et al. The ADVANCE study: a randomized study to evaluate the effects of cinacalcet plus low-dose vitamin D on vascular calcification in patients on hemodialysis. Nephrol Dial Transplant. 2011 Apr;26(4): 1327-39.

20. Bellasi A, Reiner M, Pétavy F, Goodman W, Floege J, Raggi P. Presence of valvular calcification predicts the response to cinacalcet: data from the ADVANCE Study. J Heart Valve Dis. 2013 (in press).

21. Bellasi A, Cozzolino M, Adragao T, et al. Phosphate binders in moderate chronic kidney disease: where do we stand? J Nephrol. 2013 Mar 25:0. [Epub ahead of print].

22. Bellasi A. [Hyperphosphatemia in moderate CKD: a paradox of modern nephrology] [article in Italian]. G Ital Nefrol. 2012;29:519.

23. Dhingra R, Sullivan LM, Fox CS, et al. Relations of serum phosphorus and calcium levels to the incidence of cardiovascular disease in the community. Arch Intern Med. 2007; 167(9):879-885.

24. Cozzolino M, Galassi A, Apetrii M, et al. What would we like to know, and what do we not know about fibroblast growth factor 23? J Nephrol. 2011;24:696-706.

25. Dominguez JR, Shlipak MG, Whooley MA, et al. Fractional excretion of phosphorus modifies the association between fibroblast growth factor-23 and outcomes. J Am Soc Nephrol. 2013;24:647-654.

26. Shalhoub V, Shatzen EM, Ward SC, et al. FGF23 neutralization improves chronic kidney disease-associated hyperparathyroidism yet increases mortality. J Clin Invest. 2012; 122:2543-2553.
27. Ketteler M, Biggar PH, Liangos O. FGF23 antagonism: the thin line between adaptation and maladaptation in chronic kidney disease. Nephrol Dial Transplant. 2013;28:821-825.

28. Chue CD, Townend JN, Moody WE, et al. Cardiovascular effects of sevelamer in stage 3 CKD. J Am Soc Nephrol. 2013 Apr;24(5):842-52. [Epub ahead of print].

29. Block GA, Wheeler DC, Persky MS, et al. Effects of phosphate binders in moderate CKD. J Am Soc Nephrol. 2012; 23:1407-1415.

30. Yilmaz MI, Sonmez A, Saglam M, et al. Comparison of calcium acetate and sevelamer on vascular function and fibroblast growth factor 23 in CKD patients: a randomized clinical trial. Am J Kidney Dis. 2012;59:177-185.

31. Caglar K, Yilmaz MI, Saglam M, et al. Short-term treatment with sevelamer increases serum fetuin-a concentration and improves endothelial dysfunction in chronic kidney disease stage 4 patients. Clin J Am Soc Nephrol. 2008;3:61-68.

32. Di lorio B, Bellasi A, Russo D. Mortality in kidney disease patients treated with phosphate binders: a randomized study. Clin J Am Soc Nephrol. 2012;7:487-493.

33. Di Lullo L, Floccari F, Santoboni A, et al. Progression of cardiac valve calcification and decline of renal function in CKD patients. J Nephrol. In press.

34. Cozzolino M, Mazzaferro S, Brandenburg V. The treatment of hyperphosphataemia in CKD: calcium-based or calcium-free phosphate binders? Nephrol Dial Transplant. 2011;26:402-407.

35. Bolland MJ, Avenell A, Baron JA, et al. Effect of calcium supplements on risk of myocardial infarction and cardiovascular events: meta-analysis. BMJ. 2010 Jul 29;341:c3691.

36. Bolland MJ, Barber PA, Doughty RN, et al. Vascular events in healthy older women receiving calcium supplementation: randomised controlled trial. BMJ. 2008;336:262-266.

37. Bolland MJ, Grey A, Avenell A, et al. Calcium supplements with or without vitamin $D$ and risk of cardiovascular events: reanalysis of the Women's Health Initiative limited access dataset and meta-analysis. BMJ. 2011 Apr 19;342:d2040.

38. Spiegel DM, Brady K. Calcium balance in normal individuals and in patients with chronic kidney disease on low- and high-calcium diets. Kidney Int. 2012;81:1116-1122.

39. Rodriguez-Ortiz ME, Lopez I, Munoz-Castaneda JR, et al. Calcium deficiency reduces circulating levels of FGF23. J Am Soc Nephrol. 2012;23:1190-1197.

Accepted: April 30, 2013 